\title{
Common Large Innovations Across Nonlinear Time Series
}

\author{
Richard Paap* \\ Econometric Institute \\ Erasmus University Rotterdam \\ and \\ Philip Hans Franses \\ Econometric Institute \\ Erasmus University Rotterdam \\ Econometric Institute Report 2002-09
}

\begin{abstract}
We propose a multivariate nonlinear econometric time series model, which can be used to examine if there is common nonlinearity across economic variables. The model is a multivariate censored latent effects autoregression. The key feature of this model is that nonlinearity appears as separate innovation-like variables. Common nonlinearity can then be easily defined as the presence of common innovations. We discuss representation, inference, estimation and diagnostics. We illustrate the model for US and Canadian unemployment and find that US innovation variables have an effect on Canadian unemployment, and not the other way around, and also that there is no common nonlinearity across the unemployment variables.
\end{abstract}

Key words: Nonlinearity, common features, censored latent effects autoregression

\footnotetext{
${ }^{*}$ Corresponding author: Erasmus University Rotterdam, Econometric Institute (H11-2), P.O. Box 1738, NL-3000 DR Rotterdam, the Netherlands, e-mail: paap@few.eur.nl. We thank Dennis Fok for helpful discussions. The first author thanks the Netherlands Organization for Scientific Research for its financial support.
} 


\section{Introduction}

It is now widely accepted that nonlinear time series models can capture cyclical fluctuations in economic time series variables. There are by now many such models, see for example the surveys in Granger and Teräsvirta (1993) and Franses and van Dijk (2001), among others. Examples of popular models are the (smooth) threshold autoregressions, Markov switching models, and artificial neural networks.

In many instances the nonlinear models are considered for univariate time series. This may be due to the potentially large number of parameters involved in straightforward multivariate versions of the above models. It may therefore be relevant to examine if two or more time series have common nonlinear features. An additional and economic motivation for this examination is that this could give insights as to whether two or more time series display common cyclical fluctuations.

Testing for common nonlinearity is often not an easy exercise. The main reason for this is that most nonlinear econometric time series models incorporate the nonlinearity in the parameters. For example, the smooth transition autoregression basically assumes that the autoregressive parameters are varying over time, see Granger and Teräsvirta (1993), among others. Hence, when one aims to examine common nonlinearity, in a sense one has to look for common varying parameters. Anderson and Vahid (1998) propose a method to do so, but the resulting method is based on linear regression models, which only to some extent approximate the original nonlinear model.

In this paper we propose to circumvent the above noted problems by considering a new nonlinear time series model, which basically assumes that nonlinearity can be summarized by an additional innovation-type variable. The univariate version of this, so-called, censored latent effects autoregression [CLEAR], is put forward in Franses and Paap (2002). In words, this model consists of an autoregression with two error terms. The first error term is a standard white noise process, while the second error term only once in a while gets non-zero values, depending on the outcome of a censored regression model. In Franses and Paap (2002) the CLEAR model is applied to US unemployment, where 
unexpected large positive increases are explained by a censored regression with a single leading indicator variable. Once the right-hand side of this censored regression exceeds a stochastic threshold level, a positive innovation is added to the autoregressive model. Together, the CLEAR model can capture the nonlinear features in US unemployment very well, while the number of parameters is rather small. As the nonlinear feature enters the model through a variable, the CLEAR model seems well suited for investigating common linearity. Indeed, when a linear combination of two or more variables cancels these additional variables, one has encountered common nonlinearity.

The outline of our paper is as follows. In Section 2 we outline the basics of the univariate CLEAR model and a suitable representation of its multivariate version. In Section 3, we briefly discuss unconditional inference. In Section 4, we treat parameter estimation, which can be done by Maximum Likelihood, and we deal with conditional inference. In Section 5, we illustrate our model for US and Canadian unemployment. We find that US innovation variables have an effect on Canadian unemployment, and not the other way around, and also that there is no common nonlinearity across the unemployment variables. In Section 6, we conclude with some remarks.

\section{CLEAR models}

In this section we discuss various features of the univariate CLEAR model, and a suitable representation of its multivariate extension.

\subsection{A Univariate CLEAR model}

Franses and Paap (2002) propose a censored latent effects autoregressive [CLEAR] model to describe the salient features of US unemployment. A univariate CLEAR model of order $p$ for a univariate time series $\left\{y_{t}\right\}_{t=1}^{T}$ is given by

$$
y_{t}=\sum_{i=1}^{p} \alpha_{i} y_{t-i}+x_{t}^{\prime} \gamma+v_{t}+\varepsilon_{t},
$$

where $\varepsilon_{t} \sim \operatorname{NID}\left(0, \sigma_{\varepsilon}^{2}\right), y_{p-1}, \ldots, y_{1}$ are fixed, and where $x_{t}$ is a $(k \times 1)$ vector of exogenous variables including an intercept. The variable $v_{t}$ is added as an extra innovation 
which helps to describe the sharp rise in unemployment, which is usually associated with recessions. Therefore, $v_{t}$ is imposed to be a censored variable modelled by

$$
v_{t}= \begin{cases}x_{t}^{\prime} \beta+u_{t} & \text { if } x_{t}^{\prime} \beta+u_{t} \geq 0 \\ 0 & \text { if } x_{t}^{\prime} \beta+u_{t}<0\end{cases}
$$

with $u_{t} \sim \operatorname{NID}\left(0, \sigma_{u}^{2}\right)$ and where $\beta$ and $\gamma$ are $(k \times 1)$ parameter vectors. The disturbances $\varepsilon_{t}$ and $u_{t}$ are assumed to be uncorrelated, although various extensions are possible. Also applications other than unemployment may require other versions of (2).

The effects of the exogenous variables on $y_{t}$ are given by $\gamma$ when $v_{t}=0$, while $\gamma+\beta$ represent the effects of the exogenous variables when $v_{t} \geq 0$. Hence, the CLEAR model is a nonlinear model as it allows for a time-varying effect of the explanatory variables. The nonlinear part of the model concerns the variable $v_{t}$. As (2) implies that only positive values of $v_{t}$ are added to the error term, the above CLEAR $(p)$ model contains an explicit description of what might be viewed as an exogenous positive innovation outlier generating mechanism. The variable $v_{t}$ is zero unless $x_{t}^{\prime} \beta$ exceeds a stochastic threshold level $-u_{t}$, where $u_{t}$ is a normal random variable. When the threshold is exceeded, $v_{t}$ takes a positive value.

Franses and Paap (2002) successfully describe the US unemployment rate using for $x_{t}$ a lagged value of a single leading indicator variable. In their application it turns out that the $\gamma$ may be restricted to be zero, but that the censored variable $v_{t}$ cannot be removed from the model. Graphs of the estimates of $v_{t}$ show that the innovation outlier generating variable closely follows the cyclical pattern in US unemployment.

\section{$2.2 \quad$ A Multivariate CLEAR model}

The univariate CLEAR model (1) and (2) can be generalized to a multivariate CLEAR. For example, a bivariate $\operatorname{CLEAR}(p)$ for the vector time series $\left(y_{1 t}, y_{2 t}\right)^{\prime}, t=1, \ldots, T$ may be represented by

$$
\left(\begin{array}{l}
y_{1 t} \\
y_{2 t}
\end{array}\right)=\sum_{i=1}^{p} A_{i}\left(\begin{array}{l}
y_{1 t-i} \\
y_{2 t-i}
\end{array}\right)+\left(\begin{array}{ll}
x_{1 t}^{\prime} \gamma_{11} & x_{2 t}^{\prime} \gamma_{12} \\
x_{1 t}^{\prime} \gamma_{21} & x_{2 t}^{\prime} \gamma_{22}
\end{array}\right)+\left(\begin{array}{cc}
1 & \delta_{12} \\
\delta_{21} & 1
\end{array}\right)\left(\begin{array}{l}
v_{1 t} \\
v_{2 t}
\end{array}\right)+\left(\begin{array}{l}
\varepsilon_{1 t} \\
\varepsilon_{2 t}
\end{array}\right),
$$

where $\varepsilon_{t}=\left(\varepsilon_{1 t}, \varepsilon_{2 t}\right)^{\prime} \sim \operatorname{NID}\left(\mathbf{0}, \Sigma_{\varepsilon}\right)$ with $\Sigma_{\varepsilon}$ a $(2 \times 2)$ covariance matrix, and where $A_{i}$, $i=1, \ldots, p$, are $(2 \times 2)$ parameter matrices containing the autoregressive parameters. The 
$x_{i t}$ variables are $\left(k_{i} \times 1\right)$ vectors of exogenous stationary variables including an intercept. The two innovation outlier mechanisms are modelled by

$$
v_{i t}= \begin{cases}x_{i t}^{\prime} \beta_{i}+u_{i t} & \text { if } x_{i, t}^{\prime} \beta_{i}+u_{i, t} \geq 0 \\ 0 & \text { if } x_{i, t}^{\prime} \beta_{i}+u_{i, t}<0\end{cases}
$$

where $u_{i t} \sim \operatorname{NID}\left(\mathbf{0}, \sigma_{u_{i}}^{2}\right)$ for $i=1,2$, and where $\beta_{i}, \gamma_{i i}, \gamma_{i 1}, \gamma_{2 i}$, are $\left(k_{i} \times 1\right)$ parameter vectors. The $\delta_{12}$ and $\delta_{21}$ parameters model the cross-effect of the $v_{i t}$ variables on the series $y_{t}$, and therefore we can impose that $\mathrm{E}\left[u_{1 t} u_{2 t}\right]=0$. Note that this bivariate model would be suitable for modeling unemployment series again. Other applications of the model may require other variations of (4).

\section{Common Large Innovations}

The key feature of our bivariate CLEAR model, which makes it distinct from alternative multivariate nonlinear models, advocated in for example Philips (1991), Krolzig (1997), Diebold and Rudebusch (1996) and Kim and Nelson (1998), is that we introduce nonlinearity in $y_{1 t}$ and $y_{2 t}$ through an innovation-type variable. When this variable is absent, the model is linear. This feature makes it well-suited for studying common nonlinearity.

There are several interesting restricted versions of (3) and (4), which somehow concern common properties across the two time series $y_{1 t}$ and $y_{2 t}$. An extreme case is that $y_{1 t}$ and $y_{2 t}$ have absolutely no common nonlinear features at time $t$. This implies that the $\delta_{12}$ and $\delta_{21}$ parameters are zero.

It may also be the case that only one of the $\delta$ parameters equals zero. Then, one of the series is not affected by the innovation-type variable of the other series. It may even be the case that the series have a common outlier generating mechanism. This occurs if a linear combination of $y_{1 t}$ and $y_{2 t}$ is a linear process, while the individual series are nonlinear. From (3) it is easy to see that the process $y_{2 t}-\delta_{21} y_{1 t}$ with $\delta_{21} \neq 0$ is a linear process if $v_{2 t}=\delta_{21} v_{1 t}$. The model (3) then simplifies to

$$
\left(\begin{array}{l}
y_{1 t} \\
y_{2 t}
\end{array}\right)=\sum_{i=1}^{p} A_{i}\left(\begin{array}{l}
y_{1 t-i} \\
y_{2 t-i}
\end{array}\right)+\left(\begin{array}{ll}
x_{1 t}^{\prime} \gamma_{11} & x_{2 t}^{\prime} \gamma_{12} \\
x_{1 t}^{\prime} \gamma_{21} & x_{2 t}^{\prime} \gamma_{22}
\end{array}\right)+\left(\begin{array}{c}
1 \\
\delta_{21}
\end{array}\right) v_{1 t}+\left(\begin{array}{c}
\varepsilon_{1 t} \\
\varepsilon_{2 t}
\end{array}\right)
$$

where $v_{1 t}$ is defined as in (4). Note that also the $\delta_{12}$ disappears from the model. This model assumes a common factor, and it can be viewed to correspond with the common 
trend analysis in Stock and Watson (1988). A shock $v_{1 t}$ affects both series at the same time but the size of the shock may be different due to the $\delta_{21}$ parameter. A special case amounts to $\delta_{21}=1$, where the difference between $y_{1 t}$ and $y_{2 t}$ is a linear process. In this case the size of the shock is also equal. Note that model (5) with (4) is nested in the general model (3) with (4).

Other interesting cases concern the occurrence of the shocks. An interesting bivariate CLEAR model, which is slightly different from (3) to (4) imposes that shocks $v_{1 t}$ and $v_{2 t}$ enter the vector autoregression simultaneously. This innovation outlier mechanism is given by

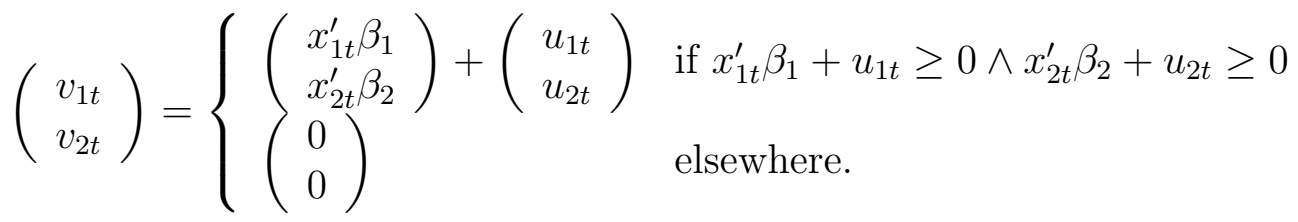

In this specification it is not possible that $v_{1 t}$ enters (3) while $v_{2 t}$ does not and vice versa. The size of the shocks may differ, of course, and therefore we do not label this model as one with common nonlinearity but one might call it synchronous nonlinearity. Note that this specification is not nested in (4), and hence it is not possible to use a standard likelihood ratio test to compare the models. Although the synchronous specification (6) seems to be a restricted version of (4), the maximum likelihood value of the model with (6) does not have to be smaller than the specification with (4). To compare the two different specifications, one can use tests for non-nested hypotheses, see for example Santos Silva (2001) and Gourieroux and Monfort (1994) for a discussion.

It is of course possible to have synchronous innovation-type variables but no common variables. In this case the model equals (3) with $\delta_{12}=\delta_{21}=0$ and with (6). The positive innovations occur at the same time but there is no relation between them. Although the synchronous model (6) is not nested in the general model (3) and (4), the common innovation outlier mechanism model (5) is nested within (3) and (6).

In the remainder of this paper we focus on estimation and inference for model specification (3) with (4) as the analysis of specification (3) with (6) can be done in a similar way. In our empirical section below we will of course compare both models. 


\section{Unconditional Inference}

The $v_{i t}$ variables in our bivariate CLEAR model are unobserved and hence we can only make probability statements about their realizations. The probability that $v_{i t}=0$ for $i=1,2$ equals the probability that $x_{i t}^{\prime} \beta_{i}+u_{i t}<0$, that is,

$$
\operatorname{Pr}\left[v_{i t}=0 \mid x_{i t} ; \theta\right]=\int_{-\infty}^{-x_{i t}^{\prime} \beta_{i}} \frac{1}{\sigma_{u_{i}}} \phi\left(u_{i t} / \sigma_{u_{i}}\right) d u_{i t}=\Phi\left(\frac{-x_{i t}^{\prime} \beta_{i}}{\sigma_{u_{i}}}\right)=\Phi_{i t},
$$

where $\theta$ summarizes the model parameters, and $\phi(\cdot)$ and $\Phi(\cdot)$ are the density function and the cumulative density function of the standard normal distribution, respectively. The probability that $v_{i t}>0$ is of course given by $\left(1-\Phi_{i t}\right)$. These probabilities can be used to predict the presence of one or two innovation-type variables at time $t$.

To predict the size of these variables, one can use the expected value of $v_{i t}$. As the $v_{i t}$ variables are censored, their expectations are not simply given by $x_{i t}^{\prime} \beta_{i}$. The expected value of the innovation-type variable $v_{i t}$ follows from

$$
\mathrm{E}\left[v_{i t} \mid x_{i t} ; \theta\right]=x_{i t}^{\prime} \beta_{i}\left(1-\Phi_{i t}\right)+\sigma_{u_{i}} \phi_{i t}, \quad \text { for } i=1,2,
$$

with $\phi_{i t}=\phi\left(-x_{i t}^{\prime} \beta_{i} / \sigma_{u_{i}}\right)$, where we have used the results on the moments of truncated normal random variables, see Johnson and Kotz (1970, p. 81-83) and Gourieroux and Monfort $\left(1995\right.$, p. 483). The variance of the variable $v_{i t}$ is equal to

$$
\mathrm{V}\left[v_{i t} \mid x_{i t} ; \theta\right]=\sigma_{u_{i}}^{2}\left(1-\Phi_{i t}\right)+x_{i t}^{\prime} \beta_{i} \mathrm{E}\left[v_{i t} \mid x_{i t} ; \theta\right]-\mathrm{E}\left[v_{i t} \mid x_{i t} ; \theta\right]^{2}
$$

for $i=1,2$, see Franses and Paap (2002) for details. Hence, the censored regressions introduce heteroskedasticity in the model.

\section{Inference}

In this section we discuss maximum likelihood estimation of the model parameters and conditional inference on the unobserved outlier mechanism. 


\subsection{Maximum Likelihood Estimation}

The model parameters of the bivariate CLEAR model (3) to (4) are summarized by $\theta=\left(A_{i}, \ldots, A_{p}, \gamma_{11}, \gamma_{12}, \gamma_{21}, \gamma_{22}, \delta_{12}, \delta_{21}, \Sigma_{\varepsilon}, \beta_{1}, \beta_{2}, \sigma_{u_{1}}^{2} \sigma_{u_{2}}^{2}\right)$. To estimate these parameters, we use a maximum likelihood estimator. For notational convenience we write (3) in matrix notation

$$
Y_{t}=\sum_{i=1}^{p} A_{i} Y_{t-i}+\Gamma X_{t}+D V_{t}+\varepsilon_{t},
$$

where $Y_{t}=\left(y_{1 t}, y_{2 t}\right)^{\prime}, X_{t}=\left(x_{1 t}^{\prime}, x_{2 t}^{\prime}\right)^{\prime}$, and $V_{t}=\left(v_{1 t}, v_{2 t}\right)^{\prime}$. The matrices $D$ and $\Gamma$ contain the $\delta$ and $\gamma$ parameters.

To derive the likelihood function, we first consider the conditional pdf of $Y_{t}$ given its past $Y^{t-1}=\left\{Y_{t-1}, \ldots, Y_{1}\right\}, X_{t}$, and given $V_{t}$. This function is given by

$$
\begin{aligned}
& f\left(Y_{t} \mid Y^{t-1}, X_{t}, V_{t} ; \theta\right)=\frac{1}{2 \pi}\left|\Sigma_{\varepsilon}\right|^{-\frac{1}{2}} \\
& \quad \exp \left(-\frac{1}{2}\left(Y_{t}-\sum_{i=1}^{p} A_{i} Y_{t-i}-X_{t} \Gamma-D V_{t}\right)^{\prime} \Sigma_{\varepsilon}^{-1}\left(Y_{t}-\sum_{i=1}^{p} A_{i} Y_{t-i}-X_{t} \Gamma-D V_{t}\right)\right) .
\end{aligned}
$$

To obtain the pdf of $Y_{t}$ conditional on $Y^{t-1}$ but unconditional on $V_{t}$, we have to integrate over the unknown error processes $u_{i t}$ in the censored regressions. The pdf of $Y_{t}$ can be decomposed in four parts corresponding to whether the $v_{i t}$ terms are zero or not

$$
f\left(Y_{t} \mid Y^{t-1}, X_{t} ; \theta\right)=g_{00}+g_{01}+g_{10}+g_{11}
$$

where

$$
\begin{aligned}
& g_{00}=\left.\operatorname{Pr}\left[v_{1 t}=0 \mid x_{1 t} ; \theta\right] \operatorname{Pr}\left[v_{2 t}=0 \mid x_{2 t} ; \theta\right] f\left(Y_{t} \mid Y^{t-1}, X_{t}, V_{t} ; \theta\right)\right|_{v_{1 t}=0, v_{2 t}=0} \\
& g_{01}=\left.\operatorname{Pr}\left[v_{1 t}=0 \mid x_{1 t} ; \theta\right] \int_{-x_{2 t}^{\prime} \beta_{2}}^{\infty} \frac{\phi\left(u_{2 t} / \sigma_{u_{2}}\right)}{\sigma_{u_{2}}} f\left(Y_{t} \mid Y^{t-1}, X_{t}, V_{t} ; \theta\right)\right|_{v_{1 t}=0, v_{2 t}=x_{2 t}^{\prime} \beta_{2}+u_{2 t}} d u_{2 t} \\
& g_{10}=\left.\operatorname{Pr}\left[v_{2 t}=0 \mid x_{2 t} ; \theta\right] \int_{-x_{1 t}^{\prime} \beta_{1}}^{\infty} \frac{\phi\left(u_{1 t} / \sigma_{u_{1}}\right)}{\sigma_{u_{1}}} f\left(Y_{t} \mid Y^{t-1}, X_{t}, V_{t} ; \theta\right)\right|_{v_{1 t}=x_{1 t}^{\prime} \beta_{1}+u_{1 t}, v_{2 t}=0} d u_{1 t} \\
& g_{11}=\left.\int_{-x_{1 t}^{\prime} \beta_{1}}^{\infty} \int_{-x_{2 t}^{\prime} \beta_{2}}^{\infty} \frac{\phi\left(u_{1 t} / \sigma_{u_{1}}\right)}{\sigma_{u_{1}}} \frac{\phi\left(u_{2 t} / \sigma_{u_{2}}\right)}{\sigma_{u_{2}}} f\left(Y_{t} \mid Y^{t-1}, X_{t}, V_{t} ; \theta\right)\right|_{v_{1 t}=x_{1 t}^{\prime} \beta_{1}+u_{1 t}, v_{2 t}=x_{2 t}^{\prime} \beta_{2}+u_{2 t}} d u_{1 t} d u_{2 t} .
\end{aligned}
$$

The log likelihood function is now simply the sum of the log of the unconditional pdfs, that is,

$$
\mathcal{L}\left(Y^{T} \mid X_{T} ; \theta\right)=\sum_{t=1}^{T} \ln f\left(Y_{t} \mid Y^{t-1}, X_{t} ; \theta\right)
$$


This log likelihood can be maximized using standard numerical optimization algorithms like Newton-Raphson. In this paper we opt for the BHHH algorithm of Berndt et al. (1974). To ensure that the covariance matrix $\Sigma_{\varepsilon}$ is positive definite we write $\Sigma_{\varepsilon}$ as $Q^{\prime} Q$ where $Q$ is a matrix with one of the off-diagonals equal to zero.

\subsection{Conditional Inference}

In Section 3 we already discussed inference on the unobserved variables in the model. This inference is unconditional on the value of $Y_{t}$ and can therefore be used in forecast exercises. In this section, we consider probabilistic inference conditional on the observed values of the time series $Y_{t}$. First, we consider the conditional probabilities that the elements of the unobserved vector $V_{t}$ equal zero. For instance, the conditional probability that $v_{1 t}=v_{2 t}=0$ given $Y_{t}$ and $x_{t}$ equals

$$
\begin{aligned}
\operatorname{Pr}\left[v_{1 t}=0 \wedge v_{2 t}=\right. & \left.0 \mid Y_{t}, X_{t} ; \theta\right]= \\
& \frac{\left.\operatorname{Pr}\left[v_{1 t}=0 \mid x_{1 t} ; \theta\right] \operatorname{Pr}\left[v_{2 t}=0 \mid x_{2 t} ; \theta\right] f\left(Y_{t} \mid Y^{t-1}, X_{t}, V_{t} ; \theta\right)\right|_{v_{1 t}=0, v_{2 t}=0}}{f\left(Y_{t} \mid Y^{t-1}, X_{t} ; \theta\right)} .
\end{aligned}
$$

The other conditional probabilities are simply

$$
\begin{aligned}
& \operatorname{Pr}\left[v_{1 t}=0 \wedge v_{2 t}>0 \mid Y_{t}, X_{t} ; \theta\right]=\frac{g_{01}}{f\left(Y_{t} \mid Y^{t-1}, X_{t} ; \theta\right)} \\
& \operatorname{Pr}\left[v_{1 t}>0 \wedge v_{2 t}=0 \mid Y_{t}, X_{t} ; \theta\right]=\frac{g_{10}}{f\left(Y_{t} \mid Y^{t-1}, X_{t} ; \theta\right)} \\
& \operatorname{Pr}\left[v_{1 t}>0 \wedge v_{2 t}>0 \mid Y_{t}, X_{t} ; \theta\right]=\frac{g_{11}}{f\left(Y_{t} \mid Y^{t-1}, X_{t} ; \theta\right)} .
\end{aligned}
$$

Hence, the marginal probabilities that $v_{1 t}=0$ and $v_{2 t}=0$ given $Y_{t}$ and $X_{t}$ equal

$$
\begin{aligned}
& \operatorname{Pr}\left[v_{1 t}=0 \mid Y_{t}, X_{t} ; \theta\right]=\operatorname{Pr}\left[v_{1 t}=0 \wedge v_{2 t}=0 \mid Y_{t}, X_{t} ; \theta\right]+\operatorname{Pr}\left[v_{1 t}=0 \wedge v_{2 t}>0 \mid Y_{t}, X_{t} ; \theta\right] \\
& \operatorname{Pr}\left[v_{2 t}=0 \mid Y_{t}, X_{t} ; \theta\right]=\operatorname{Pr}\left[v_{1 t}=0 \wedge v_{2 t}=0 \mid Y_{t}, X_{t} ; \theta\right]+\operatorname{Pr}\left[v_{1 t}>0 \wedge v_{2 t}=0 \mid Y_{t}, X_{t} ; \theta\right] .
\end{aligned}
$$

These conditional probabilities indicate whether it is likely that a positive shock affects (one of) the series at time $t$. They can be used to give a business cycle chronology, see Franses and Paap (2002) for an example. 
An estimate of the magnitude of the shock at time $t$ follows from the conditional expectation of $v_{t}$ given $Y_{t}$ and $x_{t}$, that is,

$$
\begin{aligned}
& \mathrm{E}\left[v_{1 t} \mid Y^{t}, X_{t} ; \theta\right]=\mathrm{E}[\left.v_{1 t} \mid v_{1 t}=0, Y^{t}, X_{t} ; \theta\right] \operatorname{Pr}\left[v_{1 t}=0 \mid Y^{t}, X_{t} ; \theta\right] \\
& \quad+\mathrm{E}\left[v_{1 t} \mid v_{1 t}>0, Y^{t}, X_{t} ; \theta\right] \operatorname{Pr}\left[v_{1 t}>0 \mid Y^{t}, X_{t} ; \theta\right] \\
&=\mathrm{E}\left[v_{1 t} \mid v_{1 t}>0 \wedge v_{2 t}=0, Y^{t}, X_{t} ; \theta\right] \operatorname{Pr}\left[v_{1 t}>0 \wedge v_{2 t}=0 \mid Y^{t}, X_{t} ; \theta\right] \\
& \quad+\mathrm{E}\left[v_{1 t} \mid v_{1 t}>0 \wedge v_{2 t}>0, Y^{t}, X_{t} ; \theta\right] \operatorname{Pr}\left[v_{1 t}>0 \wedge v_{2 t}>0 \mid Y^{t}, X_{t} ; \theta\right],
\end{aligned}
$$

and

$$
\begin{aligned}
\mathrm{E}\left[v_{2 t} \mid Y^{t}, X_{t} ; \theta\right]= & \mathrm{E}\left[v_{2 t} \mid v_{1 t}=0 \wedge v_{2 t}>0, Y^{t}, X_{t}\right] \operatorname{Pr}\left[v_{1 t}=0 \wedge v_{2 t}>0 \mid Y^{t}, X_{t} ; \theta\right] \\
& +\mathrm{E}\left[v_{2 t} \mid v_{1 t}>0 \wedge v_{2 t}>0, Y^{t}, X_{t} ; \theta\right] \operatorname{Pr}\left[v_{1 t}>0 \wedge v_{2 t}>0 \mid Y^{t}, X_{t} ; \theta\right] .
\end{aligned}
$$

A typical element in the expression for these conditional expectations is for instance

$$
\begin{aligned}
\mathrm{E}\left[v_{1 t} \mid v_{1 t}>0 \wedge v_{2 t}>0, Y^{t}, X_{t} ; \theta\right] & \operatorname{Pr}\left[v_{1 t}>0 \wedge v_{2 t}>0 \mid Y^{t}, X_{t} ; \theta\right] \\
=\left[f\left(Y_{t} \mid Y^{t-1}, X_{t} ; \theta\right)\right]^{-1} \int_{-x_{1 t}^{\prime} \beta_{1}}^{\infty} \int_{-x_{2 t}^{\prime} \beta_{2}}^{\infty}\left(x_{1 t}^{\prime} \beta_{1}+u_{1 t}\right) \frac{\phi\left(u_{1 t} / \sigma_{u_{1}}\right)}{\sigma_{u_{1}}} \frac{\phi\left(u_{2 t} / \sigma_{u_{2}}\right)}{\sigma_{u_{2}}} & \left.\quad f\left(Y_{t} \mid Y^{t-1}, X_{t}, V_{t} ; \theta\right)\right|_{v_{1 t}=x_{1 t}^{\prime} \beta_{1}+u_{1 t}, v_{2 t}=x_{2 t}^{\prime} \beta_{2}+u_{2 t}} d u_{1 t} d u_{2 t}
\end{aligned}
$$

and can be evaluated using the simplification in (28) in the Appendix below and the results in Rosenbaum (1961) or Maddala (1983, p. 368) concerning the expectation of a truncated bivariate normal random variable. The same holds for the other expectations in (18) and (19).

\section{Residuals}

Finally, to analyze the fit of the model, one may consider residuals. Residuals are defined

as the difference between $Y_{t}$ and the expectation of $Y_{t}$ given $Y^{t-1}$ and $X_{t}$ evaluated in the parameter estimates. This expectation equals

$$
\mathrm{E}\left[Y_{t} \mid X_{t} ; \hat{\theta}\right]=\sum_{i=1}^{p} \hat{A}_{i} Y_{t-i}+X_{t} \hat{\Gamma}+\hat{D} \mathrm{E}\left[V_{t} \mid X_{t} ; \hat{\theta}\right]
$$

where the unconditional expectation of $V_{t}$ is given in (8) for $i=1,2$. Note that the result in (9) implies that the residuals are heteroskedastic. 


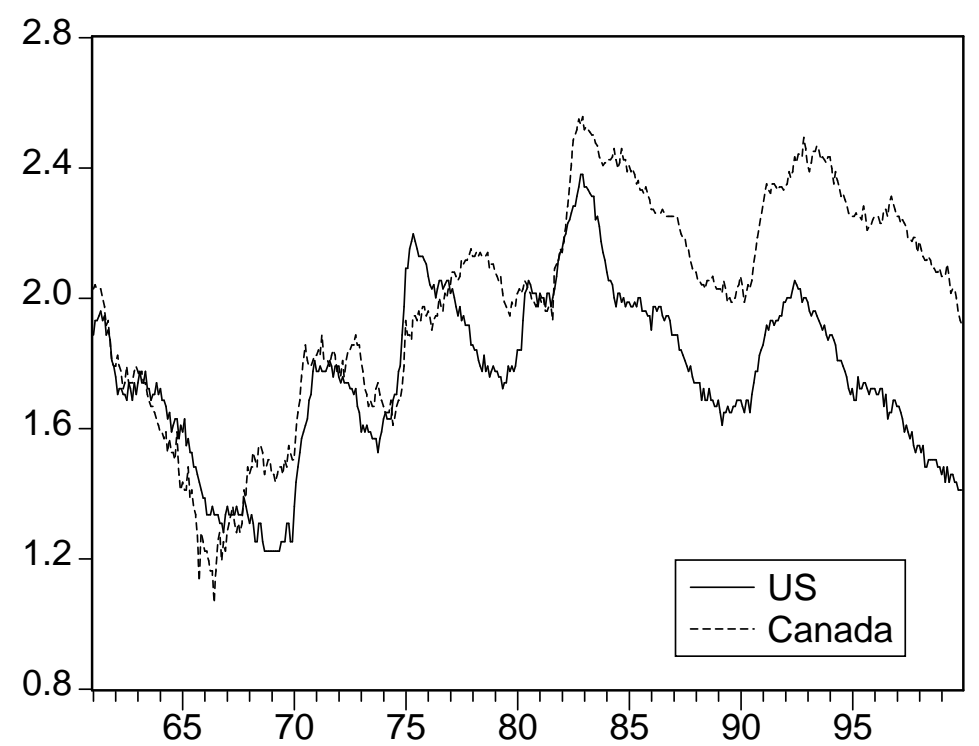

Figure 1: The logarithm of US and Canadian civilian unemployment rate, 1961.01-1999.12

\section{Unemployment in the US and Canada}

To illustrate our bivariate CLEAR model, we consider an application to seasonally adjusted monthly observed civilian unemployment rates of the United States [US] and Canada, 1961.01-1999.12. The results in Franses and Paap (2002) suggest that a univariate CLEAR is suitable for describing the US series, and hence our choice for a multivariate illustration. Figure 1 shows a graph of the natural log of both unemployment rate series. For both series we notice short periods characterized by large increases in unemployment, which are usually associated with recessions, and longer periods with a slow decline in the unemployment rate, which might match with expansions. The recessionary periods in both series seem to occur roughly in the same periods, except for the period between 1975 and 1980.

To describe both series, we consider the bivariate CLEAR model (3) and (4) for $i=1,2$. We examine whether the large increases in unemployment can be captured by two censored latent variables $v_{1 t}$ and $v_{2 t}$. Following Franses and Paap (2002), we use, as explanatory variables for the censored latent effects, the lagged values of leading indicator 


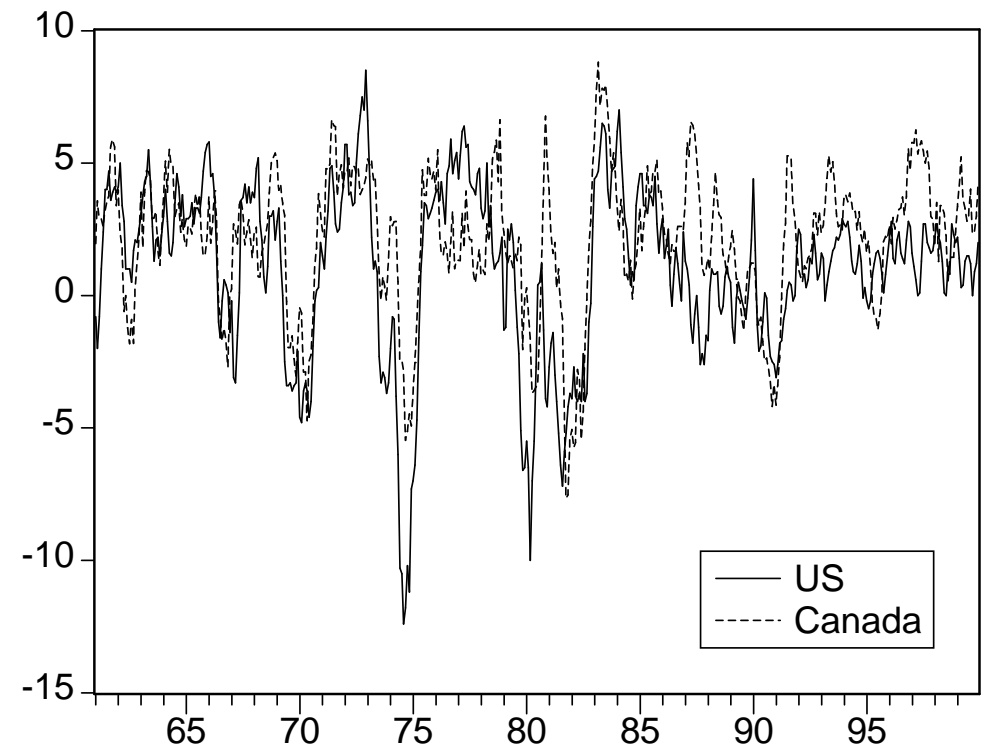

Figure 2: The US leading indicator US (XLI-2) and the bi-annual growth percentages of the Canadian composite leading indicator, 1961.01-1999.12

variables. Their results suggest that for the US the experimental leading indicator XLI- $2_{t}$ of Stock and Watson (1993) is suitable. For the Canadian series, we use the composite leading indicator series of Canada denoted by $\mathrm{CLI}_{t}$, which is obtained from Datastream. This $\mathrm{CLI}_{t}$ series display exponential growth and has to be transformed to become approximately stationary. Based on our experience in Franses and Paap (2002), we opt for the bi-annual growth rate of $\mathrm{CLI}_{t}$ denoted as $\Delta_{6} \log \mathrm{CLI}_{t}$. Figure 2 displays the two leading indicator series, which clearly bear resemblance.

To estimate our bivariate CLEAR model, we need the order $p$ and appropriate lags of the leading indicator variables. To determine this lag structure, we consider a VARX $(p)$ model which is equal to (3) with $v_{1 t}=v_{2 t}=0$. We allow for lag orders up to 12 and we consider for each lag order the appropriate lag structure of the indicator variables. It turns out that for all values of $p, \mathrm{XLI}-2_{t-1}$ and $\Delta_{6} \log \mathrm{CLI}_{t-2}$ produce the smallest determinant of the residual covariance matrix. Given this lag structure for the exogenous variables, the subsequent $\mathrm{BIC}$ value indicates that an $\mathrm{AR}$ order of 2 is best. 
We estimate a bivariate CLEAR model of order 2, where $Y_{t}$ consist of log unemployment rate of the US and Canada, respectively. The ML parameter estimates, with White's (1980) heteroskedastic-consistent standard errors in parentheses, are found to be

$$
\begin{aligned}
Y_{t}= & \left(\begin{array}{c}
0.028 \\
(0.001) \\
-0.014 \\
(0.011)
\end{array}\right)+\left(\begin{array}{cc}
0.759 & -0.064 \\
(0.046) & (0.050) \\
0.056 & 0.844 \\
(0.057) & (0.063)
\end{array}\right) Y_{t-1}+\left(\begin{array}{cc}
0.230 & 0.067 \\
(0.045) & (0.039) \\
-0.046 & 0.153 \\
(0.057) & (0.062)
\end{array}\right) Y_{t-2} \\
& +\left(\begin{array}{cc}
-0.329 & -0.250 \\
(0.072) & (0.077) \\
-0.021 & -0.142 \\
(0.098) & (0.086)
\end{array}\right)\left(\begin{array}{c}
100 \times \mathrm{XLI}_{-2} 2_{t-1} \\
\Delta_{6} \log \mathrm{CLI}_{t-2}
\end{array}\right)+\left(\begin{array}{cc}
1 & 0.364 \\
0.767 & (0.225) \\
(0.163) & 1
\end{array}\right) V_{t}+\varepsilon_{t}
\end{aligned}
$$

with

$$
\Sigma_{\varepsilon}=\frac{1}{1000}\left(\begin{array}{ll}
0.588 & 0.115 \\
0.115 & 0.491
\end{array}\right)
$$

and

$$
\begin{array}{rrr}
v_{1 t}=-\underset{(0.014)}{0}-073 & -2.355 \text { XLI- } 2_{t-1} \times 100+u_{1 t}, & u_{1 t} \sim \operatorname{NID}\left(0,0.050^{2}\right) \\
v_{2 t}=-\underset{(0.012)}{0.005}-\underset{(0.201)}{0.706} \Delta_{6} \log \mathrm{CLI}_{t-2}+u_{2 t}, & u_{2 t} \sim \operatorname{NID}\left(0,0.028^{2}\right)
\end{array}
$$

The maximum log likelihood value of this model equals 2048.66.

To check whether the lag order selected by BIC is sufficient, we also estimate a model of order 3. The maximum likelihood value of this model equals 2051.55 and the corresponding LR statistic equals 5.78. This statistic is not significant when compared with the 95 percentile of a $\chi^{2}(4)$ distribution and hence the lag order of 2 does not seem underspecified. The LR statistic for the significance of the second order autoregressive parameters equals 62.89 and hence the model cannot be simplified to a first order model.

As the order of the estimated bivariate CLEAR model seems satisfactory, we try to analyze whether the estimated model can be simplified into a model with common aspects. First, we test whether we may delete the leading indicator variables from the VAR part (22), that is, we test whether the four $\gamma$ parameters are zero. The LR statistic equals $-2 \times(2029.79-2048.66)=37.74$, which is significant compared to the $95 \%$ percentile of a $\chi^{2}(4)$ distribution. Hence we cannot delete the two indicator variables from (22). 
To analyze the role of the innovation generating censored regression, we test whether the Canadian shocks $\left(v_{2 t}\right)$ have no effect on US unemployment. The LR test for $\delta_{12}=0$ equals $-2 \times(2047.06-2048.66)=3.20$ and hence this restriction seems valid. The LR test for $\delta_{21}=0$ equals $-2 \times(2039.99-2048.66)=17.34$ and hence the innovation outlier mechanism of the US seems to affect Canadian unemployment. The joint restriction $\delta_{12}=\delta_{21}=0$ is also rejected. In words, the cyclical pattern in Canadian unemployment bears similarities with that in the US, but often not the other way around.

Fixing $\delta_{12}=0$, we may test for the presence of a common innovation outlier mechanism which corresponds to $v_{2 t}=0$. The LR statistic for $\beta_{2}=0$ and $\sigma_{u_{2}}^{2}=0$ equals $-2 \times$ $(2024.47-2047.06)=45.18$. As the total restriction contains a single one-sided alternative $\left(\sigma_{u_{2}}^{2}=0\right.$ versus $\left.\sigma_{u_{2}}^{2}>0\right)$ and two two-sided alternatives $(\beta=0$ versus $\beta \neq 0)$, this test statistic is asymptotically $\frac{1}{2} \chi^{2}(2)+\frac{1}{2} \chi^{2}(3)$ distributed, see Wolak (1989, p. 19-20). The $95 \%$ percentile of this mixture distribution is 7.80 and hence we reject the presence of a common innovation outlier mechanism.

Finally, we consider a CLEAR model with a nonlinear synchronous cycle, that is, (3) with (6). Remember that this model is not nested in the general bivariate CLEAR specification estimated in (22) and (24) and hence a standard LR test does not apply. The value of the maximum log likelihood value equals 2036.86 and it is smaller than for the model (3) with (24). The nonlinear synchronous cycle representation is not preferred.

In sum, the test results suggest that there is no common outlier mechanism, but that the outlier mechanism of Canada does not affect the US series. The parameter estimates of the final model supported by our test results are

$$
\begin{aligned}
Y_{t}= & \left(\begin{array}{c}
0.024 \\
(0.001) \\
-0.014 \\
(0.014)
\end{array}\right)+\left(\begin{array}{cc}
0.769 & -0.052 \\
(0.046) & (0.040) \\
0.058 & 0.857 \\
(0.014) & (0.069)
\end{array}\right) Y_{t-1}+\left(\begin{array}{cc}
0.220 & 0.056 \\
(0.046) & (0.039) \\
-0.048 & 0.141 \\
(0.012) & (0.069)
\end{array}\right) Y_{t-2} \\
& +\left(\begin{array}{cc}
-0.327 & -0.306 \\
(0.071) & (0.054) \\
-0.028 & -0.178 \\
(0.124) & (0.133)
\end{array}\right)\left(\begin{array}{cc}
100 \times \mathrm{XLI}_{-2} \\
\Delta_{6} \log \mathrm{CLI}_{t-2}
\end{array}\right)+\left(\begin{array}{cc}
1 & 0 \\
0.815 & 1 \\
(0.239)
\end{array}\right) V_{t}+\varepsilon_{t}
\end{aligned}
$$



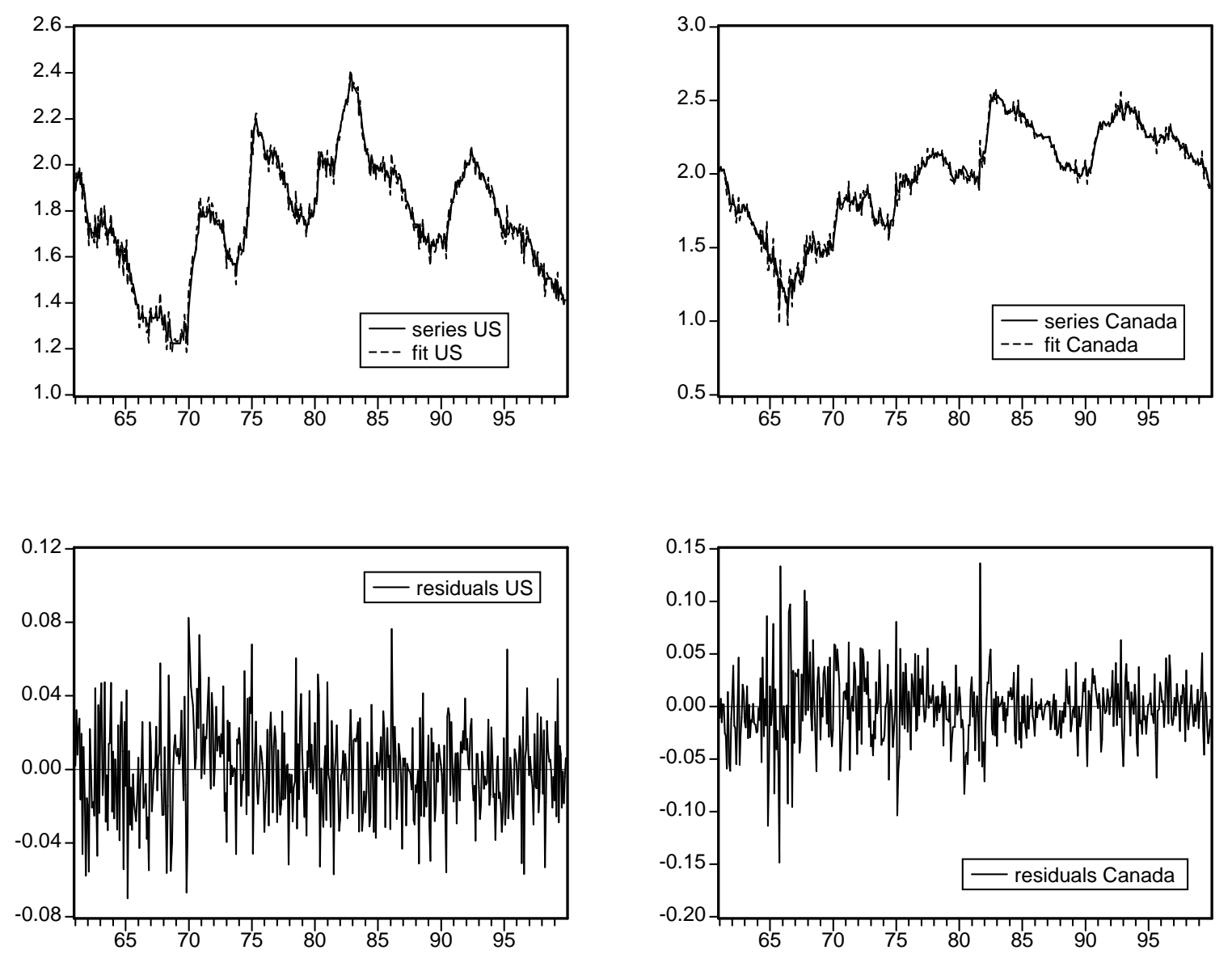

Figure 3: Fit and residuals based on (21)

with

$$
\Sigma_{\varepsilon}=\frac{1}{1000}\left(\begin{array}{ll}
0.600 & 0.114 \\
0.115 & 0.504
\end{array}\right),
$$

and

$$
\begin{aligned}
& v_{1 t}=-0.064-2.278 \text { XLI- } 2_{t-1} \times 100+u_{1 t}, \quad u_{1 t} \sim \operatorname{NID}\left(0,0.047^{2}\right) \\
& (0.022) \quad(0.527) \\
& v_{2 t}=-0.010-0.672 \Delta_{6} \log \mathrm{CLI}_{t-2}+u_{2 t}, \quad u_{2 t} \sim \operatorname{NID}\left(0,0.032^{2}\right) \quad \\
& (0.020) \quad(0.221)
\end{aligned}
$$

where again heteroskedastic-consistent standard errors appear in parentheses.

In Figure 3 we display the fit and the estimated residuals of the final model. Evidently, and even with not too many parameters, the multivariate CLEAR model provides 

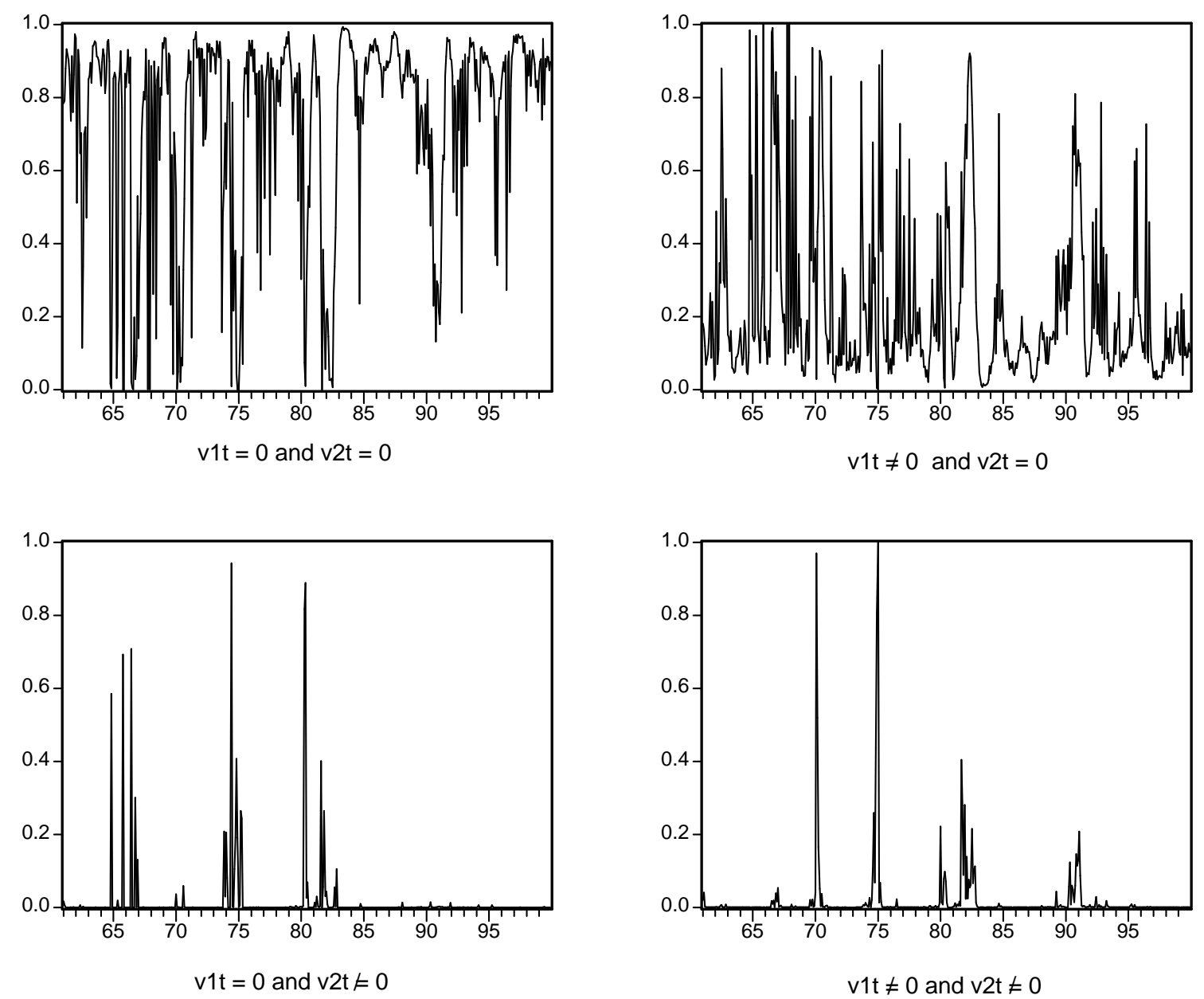

Figure 4: Estimated conditional probabilities $\operatorname{Pr}\left[v_{1 t} \wedge v_{2 t} \mid Y^{t}, X_{t}, \hat{\theta}\right]$

an excellent fit. As there are no obvious large residuals around the sharp increases in unemployment, the bivariate CLEAR model with the leading indicator variables seems to predict the start of recessionary periods rather well.

In Figure 4, we depict the estimated conditional probabilities. The left upper graph gives the probability that there are no positive innovations to both series, while the right bottom graph concern the probability that both variables both have large and positive innovations. These graphs show the familiar cyclical patterns around the oil crises and the recession in the 90s. The left bottom graph suggests that additional to the large shocks, the US only suffered from a few shocks which did not hit Canada, while the top 

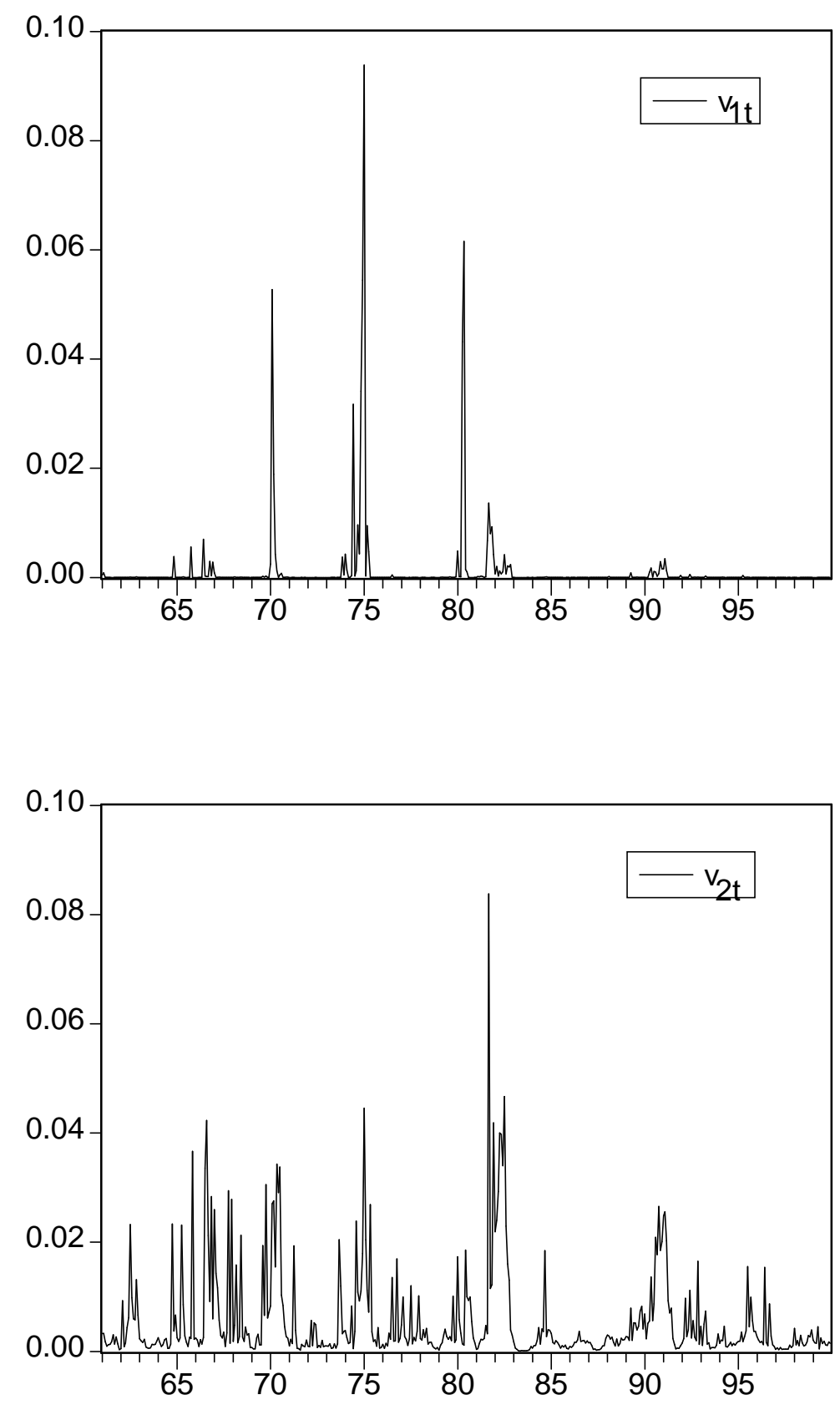

Figure 5: $\mathrm{E}\left[v_{1 t} \mid Y^{t}, X_{t}, \hat{\theta}\right]$ and $\mathrm{E}\left[v_{2 t} \mid Y^{t}, X_{t}, \hat{\theta}\right]$ 
right graph shows that Canada display much more idiosyncratic variation.

Finally, Figure 5 gives the estimated conditional expectations of the innovations. The top panel suggests that the increases in US unemployment were largest around the first oil crisis and relatively minor during the Iraq invasion in Kuwait. For Canada we observe rather different results, see the bottom panel of Figure 5, as here the second oil crisis clearly generated the largest positive shocks to unemployment.

\section{Concluding Remarks}

In this paper we put forward a new multivariate nonlinear time series model, which has the promising feature that it facilitates the analysis of common nonlinearity. This convenience is due to the fact that the model includes nonlinearity as additional variables. When linear combinations of these variables do not have such an innovation variable, these series share their nonlinearity. The model actually described nonlinear data very well, as could be seen from the illustration to US and Canadian unemployment. The illustration further suggested that nonlinear features of the US economy get reflected through Canadian unemployment, but not the other way around.

Even though the present paper only considered a bivariate illustration, it could already be seen that the multivariate censored latent autoregression is rather parsimonious. This seems a relevant feature when examining more than two time series. Furthermore, a smaller amount of parameters may also increase forecasting accuracy. We leave both issues for further research. 


\section{A Appendix}

To simplify the computation of the three integrals in the log likelihood we use the following general result

$$
\begin{aligned}
u^{\prime} \Sigma_{u}^{-1} u+(S u & -a)^{\prime} \Sigma_{\varepsilon}^{-1}(S u-a) \\
& =u^{\prime} \Sigma_{u}^{-1} u^{\prime}+u^{\prime} S^{\prime} \Sigma_{\varepsilon}^{-1} S u-a^{\prime} \Sigma_{\varepsilon}^{-1} S u-u^{\prime} S^{\prime} \Sigma_{\varepsilon}^{-1} a+a^{\prime} \Sigma_{\varepsilon}^{-1} a \\
& =u^{\prime}\left(\Sigma_{u}^{-1}+S^{\prime} \Sigma_{\varepsilon}^{-1} S\right) u+a^{\prime} \Sigma_{\varepsilon}^{-1} S u-u^{\prime} S^{\prime} \Sigma_{\varepsilon}^{-1} a+a^{\prime} \Sigma_{\varepsilon}^{-1} a \\
& =(u-b)^{\prime}\left(\Sigma_{u}^{-1}+S^{\prime} \Sigma_{\varepsilon}^{-1} S\right)(u-b)-b^{\prime}\left(\Sigma_{u}^{-1}+S^{\prime} \Sigma_{\varepsilon}^{-1} S\right) b+a^{\prime} \Sigma_{\varepsilon}^{-1} a
\end{aligned}
$$

with $b=\left(\Sigma_{u}^{-1}+S^{\prime} \Sigma_{\varepsilon}^{-1} S\right)^{-1} S^{\prime} \Sigma_{\varepsilon}^{-1} a$. This result allows us to write the product of the three normal pdfs in the three integrals which both depend on $u_{t}$ as the product of a normal pdf which depends on $u_{t}$ and a remainder that does not depend on $u_{t}$. Hence, each integral can be expressed in terms of a CDF of a bivariate normal distribution. 


\section{References}

Anderson, H. and F. Vahid (1998), Testing multiple equation systems for common nonlinear components, Journal of Econometrics, 84, 1-36.

Berndt, E., B. Hall, E. Hall, and J. Hausman (1974), Estimation and Inference in Nonlinear Structural Models, Annals of Economic and Social Measurement, 3, 653-665.

Diebold, F. and G. Rudebusch (1996), Measuring Business Cycles: A Modern Perspective, Review of Economics and Statistics, 78, 67-77.

Franses, P. and R. Paap (2002), Censored Latent Effects Autoregression, with an Application to US Unemployment, Journal of Applied Econometrics, forthcoming.

Franses, P. and D. van Dijk (2001), Nonlinear Time Series Models in Empirical Finance, Cambridge University Press, Cambridge.

Gourieroux, C. and A. Monfort (1994), Testing Non-Nested Hypotheses, in R. Engle and D. McFadden (eds.), Handbook of Econometrics, vol. IV, chap. 44, North-Holland, Amsterdam, pp. 2583-2637.

Gourieroux, C. and A. Monfort (1995), Statistics and Econometric Models, vol. 2, Cambridge University Press, Cambridge.

Granger, C. and T. Teräsvirta (1993), Modelling Nonlinear Economic Relations, Oxford University Press, Oxford.

Johnson, N. and S. Kotz (1970), Distributions in Statistics: Continuous Univariate Distributions, Houghton Mifflin, Boston.

Kim, C.-J. and C. Nelson (1998), Business Cycle Turning Points, a New Coincident Index, and Tests of Duration Dependence on a Dynamic Factor Model with Regime Switching, Review of Economics and Statistics, 80, 188-201.

Krolzig, H.-M. (1997), Markov Switching Vector Autoregressions: Modelling, Statistical Inference, and an Application to Business Cycle Inference, Springer, Berlin. 
Maddala, G. (1983), Limited Dependent and Qualitative Variables in Econometrics, vol. 3 of Econometric Society Monographs, Cambridge University Press, Cambridge.

Philips, K. (1991), A Two-Country Model for Stochastic Output with Changes in Regime, Journal of International Economics, 31, 121-142.

Rosenbaum, S. (1961), Moments of a Truncated Bivariate Normal Distribution, Journal of the Royal Statistical Society B, 23, 405-408.

Santos Silva, J. (2001), A Score Test for Non-Nested Hypotheses with Applications to Discrete Data Models, Journal of Applied Econometrics, 16, 577-597.

Stock, J. and M. Watson (1988), Testing for Common Trends, Journal of the American Statistical Association, 83, 1097-1107.

Stock, J. and M. Watson (1993), A Procedure for Predicting Recessions with Leading Indicators: Econometric Issues and Recent Experience, in J. Stock and M. Watson (eds.), Business Cycles, Indicators, and Forecasting, chap. 2, The University of Chicago Press, Chicago, pp. 95-147.

White, W. (1980), A Heteroscedasticity-consistent Covariance Matrix Estimator and a Direct Test for Heteroscedasticity, Econometrica, 48, 817-838.

Wolak, F. (1989), Local and Global Testing of Linear and Nonlinear Inequality Constraints in Nonlinear Econometric Models, Econometric Theory, 5, 1-35. 